\title{
Immunogenicity of Cpn0425 and its localization in cells infected with Chlamydophila pneumoniae
}

\author{
LIANGZHUAN LIU ${ }^{1}$, XIAOXING YOU ${ }^{1}$, LIESONG CHEN ${ }^{1}$, YANHUA ZENG ${ }^{1}$, \\ SHUANGYANG TANG ${ }^{1}$, MINJUN YU ${ }^{1}$, YIMOU WU ${ }^{1}$ and XI XHEN ${ }^{2}$ \\ ${ }^{1}$ Pathogenic Biology Institute, University of South China, Hengyang 421001; \\ ${ }^{2}$ Hunan Provincial Center of Disease Prevention and Control, Changsha 410005, P.R. China
}

Received April 25, 2012; Accepted August 21, 2012

DOI: $10.3892 / \mathrm{mmr} .2012 .1083$

\begin{abstract}
The present study aimed to determine the intracellular localization of Cpn0425 in Chlamydophila pneumoniae-infected cells. The recombinant plasmid pGEX-6p/ Cpn0425 was transformed into E.coli $\mathrm{B} 121$ cells to express the fusion protein. Following purification with glutathione S-transferase (GST) resin chromatography, the Cpn0425 fusion protein was used to induce immunity in mice to develop monoclonal and polyclonal antibodies, which were subsequently used to localize the endogenous Cpn0425 protein by indirect immunofluorescence assay (IFA). ELISA was used to determine the immunogenicity of the Cpn0425 plasmid protein by recognizing the pool sera of patients infected with Chlamydia trachomatis and the pool sera of mice immunized with the Cpn0425 fusion protein. The Cpn0425 gene was expressed as the GST-Cpn0425 fusion protein in E. coli and its antibody was prepared by immunizing mice with the fusion protein. An anti-GST-Cpn0425 antibody was used to localize the protein in cells infected with Chlamydophila pneumoniae AR-39 using an IFA. The anti-GST-CT058 antibody detected an inclusion signal in the IFA. Cpn0425 protein strongly reacted with antiserum. Although Cpn0425 protein is not a secreted protein, it has good immunogenicity. Therefore, this protein may be useful for developing vaccines against Chlamydophila pneumoniae infection.
\end{abstract}

\section{Introduction}

Chlamydophila pneumoniae ( $\mathrm{Cpn}$ ) is a pathogen that causes respiratory infections such as pneumonia, asthma, chronic pharyngitis, chronic bronchitis and cardiovascular diseases (1-5). Although its pathogenic mechanism remains unclear, Cpn may result from its toxic protein secretion. The type III

Correspondence to: Professor Yimou Wu, Pathogenic Biology Institute, University of South China, Hengyang 421001, P.R. China E-mail: yimouwu82503@yahoo.com.cn

Keywords: Chlamydophila pneumoniae, Cpn0425, cell localization, immunogenicity secretion system (T3SS) is an independent secretion system since its effector proteins may change the cytoskeleton formation, destroy the signal transduction pathways, inhibit apoptosis and interfere with transcriptional regulation (6-9). Screening and identification of the Cpn T3SS effector proteins have become a research hotspot globally (10-12). Genes encoding T3SS effector proteins are believed to be positioned close to their chaperones (13). Gene Cpn lcrH1 is Cpn homologous to Yersinia and adjacent to Cpn0425 (14,15). Since Cpn0425 is located in the T3SS-encoding gene family, it is expected to be one of the Cpn T3SS effector proteins. In this study, the Cpn0425 protein was cloned, expressed and purified in vitro, and its localization and immune activities in infected cells were investigated to further study the screening, identification and pathogenic action of Cpn T3SS effector proteins.

\section{Materials and methods}

Plasmids and reagents. The pGEX 6p-2 plasmid, E. coli $\mathrm{Bl} 21$ strains and THP-1 cell lines were provided by the Institute of Pathogenic Biology, Nanhua University (China). AxyPrep PCR kit was purchased from Axygen Biosciences (Union City, CA, USA), GST purification resin was obtained from Novagen (Madison, WI, USA) and the BCA protein concentration assay kit was purchased from Biyuntian Company (Jiangsu, China). The assay kit for the TNF- $\alpha$ and IL- 6 cytokines were provided by Jingmei Biotech Co., Ltd. (Shenzhen, China).

Construction of $p G E X-6 p-2 / C p n 0425$. The Cpn AR-39 genome was used as the template to amplify Cpn0425 coding genes by PCR. Recombinant plasmid pGEX-6p-2/Cpn0425 was transformed into vector $E$. coli $\mathrm{Bl} 21$ for enzyme digestion and sequencing.

Expression, purification and identification of recombinant proteins. Recombinant protein GST-Cpn0425 was induced by IPTG and purified by GST purification resin. Following the expression and purity analysis of $10 \%$ SDS-PAGE, the purified product was identified by western blotting.

Preparation of serum. The purified GST-Cpn0425 fusion protein was emulsified with the same amount of Freund's complete adjuvant, and intraperitoneally injected into 12 immune 6-week- 
old BALB/c mice. Fourteen days after the initial immunization, fusion protein with incomplete Freund's adjuvant was applied 3 times every 10 days to strengthen immunity. Seven days after the last immunization, a blood sample was taken. The serum was separated to obtain polyclonal antibodies. Indirect immunofluorescence assay (IFA) was performed to detect the location and immune activity of the Cpn0425 protein in Cpn-infected Hep-2 cells.

IFA for Chlamydia protein. Cell growth solution (1 ml), containing $8 \times 10^{4} \mathrm{Hep}-2$ cells, was added to each well of the 24-well tissue culture plates and cultured for $18 \mathrm{~h}$. The medium was discarded when the cells grew into a monolayer. Cells were inoculated with Cpn AR-39, followed by the addition of $2 \mathrm{mg} / \mathrm{l}$ cycloheximide solution. Cells were then incubated for $72 \mathrm{~h}$. The chlamydial infection solution was aspirated from the wells and fixed with 4\% paraformaldehyde. Rabbit anti-CT polyclonal antibody Cpn AR-39 was double-stained with dilutions (1:200, 1:1000 and 1:5000) of mouse anti-Cpn 0425 antibody, and incubated at $37^{\circ} \mathrm{C}$ for $1 \mathrm{~h}$. After washing, Cy2-labeled goat anti-rabbit IgG and Cy3-labeled goat anti-mouse $\operatorname{IgG}$ were added to the solution. The nucleus was marked by Hoechst and observed under a fluorescence microscope after incubation at $37^{\circ} \mathrm{C}$ for $1 \mathrm{~h}$ and washing.

ELISA. Savyon GST-Cpn0425 protein was diluted by $0.05 \mathrm{~mol} / \mathrm{l}, \mathrm{pH} 7.4$ carbonate buffer, and $100 \mu \mathrm{g}$ of the solution was then added to each well of the microtiter plates for the detection of Cpn standard serum. Negative and positive controls were established with tetramethylbenzidine (TMB) as the chromogenic substrate, and $\mathrm{A}_{450}$ values were measured by microplate reader. Additionally, 20 cases of Cpn-positive serum and 10 cases of Cpn-negative serum were determined by the established indirect ELISA methods. The test serum was diluted at 1:100, and a $\mathrm{A}_{450}$ value $+2 \mathrm{~S}$ was considered as the cut-off value. When the $\mathrm{A}_{450}$ value was higher than the cut-off level, the value was regarded as positive, otherwise it was negative. The results were compared with the test results of the SeroCP ${ }^{\mathrm{TM}}$ IgG ELISA diagnostic kit from Savyon Diagnostics (Ashdod, Israel) and the application value of the recombinant protein antigens in the serological diagnosis of Cpn was preliminarily evaluated.

\section{Results}

Induced expression of the recombinant plasmid pGEX6p-2/ Cpn0425.PCR products were separated on agarose gels, which showed a clear and specific band located approximately at $350 \mathrm{bp}$ (Fig. 1A). This result was consistent with the expected fragment size of the $\mathrm{Cpn} 0425$ products (588 bp).

The recombinant plasmid pGEX6p-2/Cpn0425 was transformed into the E. coli $\mathrm{Bl} 21$ host strain for induced expression. Results of the SDS-PAGE showed an obvious band of the induced protein at approximately $50 \mathrm{kDa}$. This result was consistent with the expected molecular weight of $\mathrm{Cpn} 0425$ (Fig. 1B).

Western blot analysis of the recombinant protein GST-Cpn0425. After the induced expression of $0.2 \mathrm{~mol} / \mathrm{l}$ IPTG for $4 \mathrm{~h}$, results showed that the recombinant protein was
A

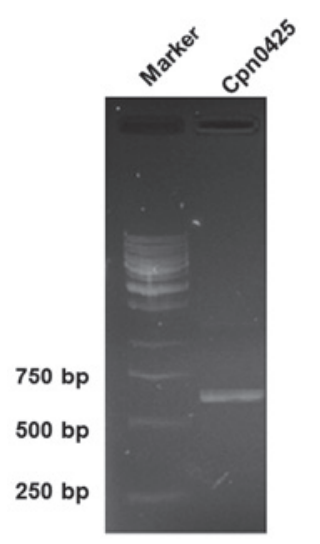

B

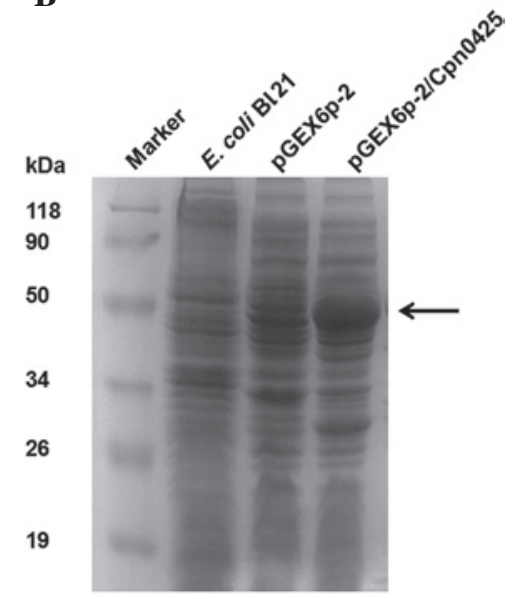

Figure 1. (A) PCR analysis of pGEX6p-2/Cpn0425 plasmid. (B) Expression of recombinant plasmid pGEX6p-2/Cpn0425 in B121 cells. M, Marker; lane 1, E. coli B121; lane 2, expression of recombinant plasmid pGEX6p-2 in B121 cells; lane 3, expression of recombinant plasmid pGEX6p-2/Cpn0425 in B121 cells.

present mainly in the supernatant, but little in the precipitation. This result indicates that pGEX6p-2/Cpn0425 was expressed mainly in soluble form, and only a little in the inclusion bodies. GST of the recombinant protein GST-Cpn0425 was purified and analysed by $12 \%$ SDS-PAGE. The purification of fusion proteins was found to have been obtained in the corresponding positions with $\mathrm{M}_{\mathrm{r}}$ of approximately $50 \mathrm{kDa}$. Bands below the fusion protein were considered as fusion protein degradation products (Fig. 2A).

Rat anti-Cpn AR39 polyclonal antibody was the primary antibody and was detected by western blot analysis for the induced expression of GST-Cpn 0425, which showed obvious specific bands, at approximately $50 \mathrm{kDa}$ of $\mathrm{M}_{\mathrm{r}}$, whereas no bands were observed of the uninduced bacteria (Fig. 2B).

Positioning of the Cpn0425 protein in infected cells. After the infection of Cpn AR-39 in Hep-2 cells for $72 \mathrm{~h}$, cells were fixed and the fusion protein polyclonal antibody serum was considered as the primary antibody. IFA results showed that the Cpn 0425 protein was present in intracellular inclusions (Fig. 3). Indirect ELISA was applied for the detection of Cpn IgG antibodies in the serum of Cpn-infected patients (Table I).

\section{Discussion}

In this experiment, the expression proteins of the clone and transformation vector pGEX-6P-2 were fusion proteins, which facilitated to further separate the purified protein. In addition, the selection of the expression bacteria highly expressed the key factors of fusion proteins (16). Expression bacteria applied in this study was E.coli Bl21, a commonly used expression strain that generally strongly expresses target genes together with a strongly expressed vector pGEX. The Bl21 strain lacks Lon and OmpT protease products, thus reducing the impact of protease degradation in the host cells. In addition, this strain helps to increase the yield of soluble protein and facilitates the efficient expression of soluble GST fusion protein. GST 
A

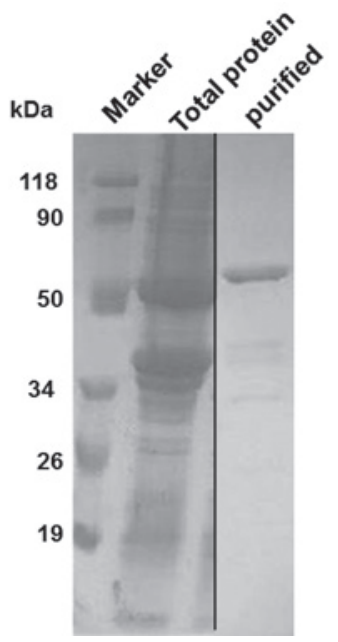

B

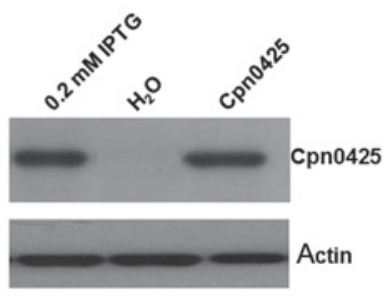

Figure 2. (A) SDS-PAGE of purified GST-Cpn0425 and the total proteins. (B) Western blot analysis of the GST-Cpn0425 recombinant protein expressed in the pGEX6p-2/Cpn0425 Bl21 cells induced with or without IPTG. The purified GST-Cpn0425 recombinant protein served as a control.

A

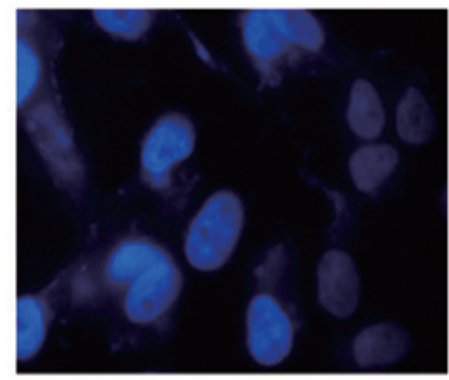

B

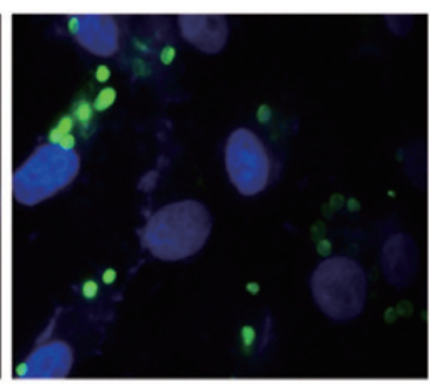

C

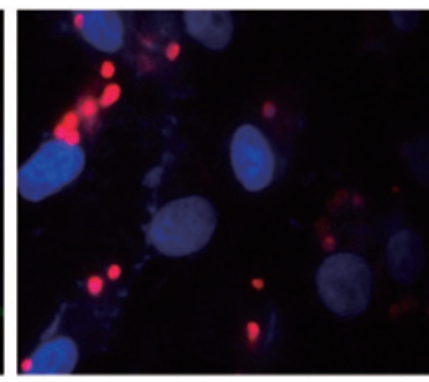

D

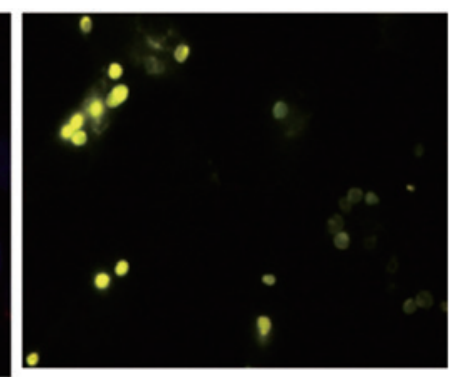

Figure 3. Localization of Cpn0425 in the Chlamydophila pneumoniae (Cpn)-infected cells. Hep-2 cells infected with Cpn were immunostained with mouse anti-GST-Cpn0425 fusion protein antibodies visualized with a goat anti-mouse IgG-conjugated Cy3 (red), a rabbit anti-Cpn AR39 visualized with a Cy2conjugated goat anti-rabbitIgG (green) and a DNA dye (blue). (A) Normal Hep-2 cells without infection. (B) The anti-Cpn AR39 antibody detected a dominant signal in the inclusion body (green fluorescence). (C) The anti-GST-Cpn0425 antibody detected a dominant signal in the inclusion body (red fluorescence). (D) Overlapped fluorescence (orange) as detected by the anti-Cpn AR39 and anti-GST-Cpn0425 antibodies.

Table I. ELISA detection of serum from the Chlamydophila pneumoniae (Cpn)-infected patients using the recombinant Cpn0425 as antigen.

\begin{tabular}{lccr}
\hline Serum of patients & \multicolumn{3}{c}{ Indirect ELISA results } \\
\cline { 2 - 4 }$(\mathrm{Cpn} \operatorname{IgG})$ & Positive & Negative & Total \\
\hline Positive & 17 & 3 & 20 \\
Negative & 0 & 10 & 10 \\
Total & 17 & 13 & 30 \\
\hline
\end{tabular}

fusion protein was purified by GST purification resin (Merck Novagen). The resin is of high specificity for the GST purification filter and of specific adsorption of the GST fusion protein, which subsequently improves the purity of the target protein. Purification is achieved through the affinity of GST and glutathione (GSH), and the purified protein obtained maintains its natural biological activity since only a natural GST protein has affinity characteristics.
Polyvalent mouse immune serum was obtained using purified recombinant protein-immunized BALB/c mice. Its high specific antibody titer was determined by indirect ELISA. This revealed the high immunogenicity of the recombinant protein-stimulated $\mathrm{BALB} / \mathrm{c}$ mice that produced a strong humoral immune response, resulting in the gradual increase of the immune effect. Due to its high immunogenicity, the fusion protein antibody obtained in this experiment may be applied in western blotting, immunofluorescence and other types of research techniques.

Seventeen out of 20 cases showed a positive reaction to the fusion protein and serum from patients with respiratory tract infections, suggesting that $\mathrm{Cpn} 0425$ may be produced in the natural infection. Since this endogenous protein exhibits immunogenicity, it stimulates the body to respond differently. Thus, Cpn0425 may be used as a new chlamydia-immune diagnostic reagent, and may even be applied in the laboratory to diagnose Cpn infection (17).

Three color marker staining was applied in the IFA staining process: DNA was stained blue by Hoechst, whole CT cell was stained green by Cy2-labeled fluorescent antibody, while target protein positioning was stained red by the Cy3-labeled 
fluorescent antibody. When target proteins were distributed around bacteria, green and red fluorescence were overlapped and the IFA results appeared to be yellow or orange. If the target proteins were at the inclusion body membrane (17-20), it was red around the inclusion for the membrane staining in IFA results. IFA results in this study showed that the Cpn0425 protein was located inside the inclusion bodies in infected cells, indicating that $\mathrm{Cpn} 0425$ was not an effector protein in the Chlamydia type III secretion system.

In conclusion, the cell localization of the CT0425 protein, present in inclusion bodies with strong immune activity, in $C$. trachomatis-infected cells was successfully desmonstrated in this study through IFA. Additional studies are required to investigate the structure and biological function of CT0425.

\section{Acknowledgements}

This project is funded by the Doctoral Fund of the Ministry of Education (200805400002) of the Health Department of the Province of Hunan (B2011-040).

\section{References}

1. Lui G, Ip M, Lee N, et al: Role of 'atypical pathogens' among adult hospitalized patients with community-acquired pneumonia Respirology 14: 1098-1105, 2009.

2. Damy SB, Higuchi ML, Timenetsky J, et al: Mycoplasma pneumoniae and/or Chlamydophila pneumoniae inoculation causing different aggravations in cholesterol-induced atherosclerosis in apoE KO male mice. BMC Microbiol 9: 194, 2009.

3. Papaetis GS, Anastasakou E and Orphanidou D: Chlamydophila pneumoniae infection and COPD: more evidence for lack of evidence? Eur J Intern Med 20: 579-785, 2009.

4. Song JH, Thamlikitkul V and Hsueh PR: Clinical and economic burden of community-acquired pneumonia amongst adults in the Asia-Pacific region. Int J Antimicrob Agents 38: 108-117, 2011.

5. Jackson RW, Vinatzer B, Arnold DL, Dorus S and Murillo J: The influence of the accessory genome on bacterial pathogen evolution. Mob Genet Elements 1: 55-65, 2011.

6. Mota LJ: Cornelis. The bacterial injection kit: type III secretion systems. Ann Med 37: 234-249, 2005

7. Koizumi Y,Toma C, Higa N, Nohara T, Nakasone N and Suzuki T: Inflammasome activation via intracellular NLRs triggered by bacterial infection. Cell Microbiol 14: 149-154, 2012.
8. Diaz MR, King JM and Yahr TL: Intrinsic and extrinsic regulation of type III secretion gene expression in pseudomonas aeruginosa. Front Microbiol 2: 89, 2011.

9. Dean P: Functional domains and motifs of bacterial type III effector proteins and their roles in infection. FEMS Microbiol Rev 35: 1100-1125, 2011.

10. Park SH, Kwon SJ, Lee SJ, Kim YC, Hwang KY, Kang YH and Lee KJ: Identification of immunogenic antigen candidate for Chlamydophila pneumoniae diagnosis. J Proteome Res 8: 2933-2943, 2009.

11. Tammiruusu A, Penttilä T, Lahesmaa R, Sarvas M, Puolakkainen $\mathrm{M}$ and Vuola JM: Intranasal administration of chlamydial outer protein $\mathrm{N}(\mathrm{CopN})$ induces protection against pulmonary Chlamydia pneumoniae infection in a mouse model. Vaccine 25: 283-290, 2007.

12. Kalman S, Mitchell W, Marathe R, et al: Comparative genomes of Chlamydia pneumoniae and C. trachomatis. Nat Genet 21: 385-389, 1999.

13. Chen CQ, Wu YM, Li ZY, et al: Chlamydia trachomatis outer membrane protein expression of recombinant protein purification and immunological identification. Chin Lab Med 28: 697-699, 2005.

14. Lugert R, Kuhns M, Polch T and Gross U: Expression and localization of type III secretion-related proteins of Chlamydia pneumoniae. Med Microbiol Immunol 193: 163-171, 2004.

15. Timms P, Good D, Wan C, Theodoropoulos C, Mukhopadhyay S, Summersgill J and Mathews S: Differential transcriptional responses between the interferon-gamma-induction and ironlimitation models of persistence for Chlamydia pneumoniae. J Microbiol Immunol Infect 42: 27-37, 2009.

16. Chen C, Chen D, Sharma J, et al: The hypothetical protein CT813 is localized in the Chlamydia trachomatis inclusion membrane and is immunogenic in women urogenitally infected with C. trachomatis. Infect Immun 74: 4826-4840, 2006.

17. Huang Z, Feng Y, Chen D, et al: Structural basis for activation and inhibition of the secreted chlamydia protease CPAF. Cell Host Microbe 4: 529-542, 2008.

18. Pirbhai M, Dong F, Zhong Y, et al: The secreted protease factor $\mathrm{CPAF}$ is responsible for degrading pro-apoptotic $\mathrm{BH} 3$-only proteins in Chlamydia trachomatis-infected cells. J Biol Chem 281: 31495-31501, 2006

19. Li ZY, Wang SP, Wu YM, et al: Immunization with chlamydial plasmid protein pORF5 DNA vaccine induces protective immunity against genital chlamydial infection in mice. Sci China C Life Sci. 51: 973-980, 2008.

20. Toh H, Miura K, Shirai M, et al: In silico inference of inclusion membrane protein family in obligate intracellular parasites chlamydiae. DNA Res 10: 9-17, 2003. 